\title{
What do we know about stem cells and application in Pharmacology
}

\author{
Ramya $K$ and Sankar $\mathbf{P}^{*}$ \\ Department of Pharmacology and Toxicology, Veterinary College and Research Institute, India
}

\section{What are stem cells, and why are they important?}

Stem cells have the remarkable potential to develop into many different cell types in the body during early life and growth. In addition, in many tissues they serve as a sort of internal repair system, dividing essentially without limit to replenish other cells as long as the person or animal is still alive. When a stem cell divides, each new cell has the potential either to remain a stem cell or become another type of cell with a more specialized function, such as a muscle cell, a red blood cell, or a brain cell.

Stem cells are distinguished from other cell types by two important characteristics. First, they are unspecialized cells capable of renewing themselves through cell division, sometimes after long periods of inactivity. Second, under certain physiologic or experimental conditions, they can be induced to become tissue- or organ-specific cells with special functions. In some organs, such as the gut and bone marrow, stem cells regularly divide to repair and replace worn out or damaged tissues. In other organs, however, such as the pancreas and the heart, stem cells only divide under special conditions.

Until recently, scientists primarily worked with two kinds of stem cells from animals and humans: embryonic stem cells and nonembryonic "somatic" or "adult" stem cells. Scientists discovered ways to derive embryonic stem cells from early mouse embryos more than 30 years ago, in 1981. The detailed study of the biology of mouse stem cells led to the discovery, in 1998, of a method to derive stem cells from human embryos and grow the cells in the laboratory. These cells are called human embryonic stem cells. The embryos used in these studies were created for reproductive purposes through in vitro fertilization procedures. In 2006, researchers made another breakthrough by identifying conditions that would allow some specialized adult cells to be "reprogrammed" genetically to assume a stem cell-like state. This new type of stem cell, called induced pluripotent stem cells (iPSCs).

Stem cells are important for living organisms for many reasons. In the 3- to 5-day-old embryo, called a blastocyst, the inner cells give rise to the entire body of the organism, including all of the many specialized cell types and organs such as the heart, lungs, skin, sperm, eggs and other tissues. In some adult tissues, such as bone marrow, muscle, and brain, discrete populations of adult stem cells generate replacements for cells that are lost through normal wear and tear, injury, or disease.

\section{What are the unique properties of all stem cells?}

Stem cells differ from other kinds of cells in the body. All stem cells, regardless of their source have three general properties: they are capable of dividing and renewing themselves for long periods; they are unspecialized; and they can give rise to specialized cell types.
- Stem cells are capable of dividing and renewing themselves for long periods.

Unlike muscle cells, blood cells, or nerve cells-which do not normally replicate themselves-stem cells may replicate many times, or proliferate. A starting population of stem cells that proliferates for many months in the laboratory can yield millions of cells. If the resulting cells continue to be unspecialized, like the parent stem cells, the cells are said to be capable of long-term self-renewal [1].

\section{- Stem cells are unspecialized}

One of the fundamental properties of a stem cell is that it does not have any tissue specific structures that allow it to perform specialized functions. For example, a stem cell cannot work with its neighbours to pump blood through the body (like a heart muscle cell), and it cannot carry oxygen molecules through the bloodstream (like a red blood cell). However, unspecialized stem cells can give rise to specialized cells, including heart muscle cells, blood cells, or nerve cells.

\section{- Stem cells can give rise to specialized cells.}

When unspecialized stem cells give rise to specialized cells, the process is called differentiation. While differentiating, the cell usually goes through several stages, becoming more specialized at each step. Scientists are just beginning to understand the signals inside and outside cells that trigger each step of the differentiation process. The internal signals are controlled by a cell's genes, which are interspersed across long strands of DNA and carry coded instructions for all cellular structures and functions. The external signals for cell differentiation include chemicals secreted by other cells, physical contact with neighbouring cells, and certain molecules in the microenvironment. The interaction of signals during differentiation causes the cell's DNA to acquire epigenetic marks that restrict DNA expression in the cell and can be passed on through cell division [2].

Adult stem cells typically generate the cell types of the tissue in which they reside. For example, a blood-forming adult stem cell in the bone marrow normally gives rise to the many types of blood cells. It is generally accepted that a blood-forming cell in the bone marrowwhich is called a hematopoietic stem cell-cannot give rise to the cells of a very different tissue, such as nerve cells in the brain. Experiments

Correspondence to: Palanisamy Sankar, Assistant Professor, Department of Pharmacology and Toxicology, Veterinary College and Research Institute, Orathanadu, Thanjavur-614625, India, Tel: +91 7598343591, E-mail: drpsankarster@gmail.com

Received: February 24, 2015; Accepted: March 20, 2015; Published: March 23, 2015 
over the last several years have purported to show that stem cells from one tissue may give rise to cell types of a completely different tissue. This remains an area of great debate within the research community. This controversy demonstrates the challenges of studying adult stem cells and suggests that additional research using adult stem cells is necessary to understand their full potential as future therapies.

\section{What are embryonic stem cells?}

Embryonic stem cells are derived from embryos that develop from eggs that have been fertilized in vitro. As long as the embryonic stem cells in culture are grown under appropriate conditions, they can remain undifferentiated (unspecialized). But if cells are allowed to clump together to form embryoid bodies, they begin to differentiate spontaneously. They can form muscle cells, nerve cells, and many other cell types. Although spontaneous differentiation is a good indication that a culture of embryonic stem cells is healthy, it is not an efficient way to produce cultures of specific cell types.

So, to generate cultures of specific types of differentiated cellsheart muscle cells, blood cells, or nerve cells, for example-scientists try to control the differentiation of embryonic stem cells. They change the chemical composition of the culture medium, alter the surface of the culture dish, or modify the cells by inserting specific genes. Through years of experimentation, scientists have established some basic protocols or "recipes" for the directed differentiation of embryonic stem cells into some specific cell types [1,2].

\section{What are adult stem cells?}

An adult stem cell is thought to be an undifferentiated cell, found among differentiated cells in a tissue or organ. The adult stem cell can renew itself and can differentiate to yield some or all of the major specialized cell types of the tissue or organ. The primary roles of adult stem cells in a living organism are to maintain and repair the tissue in which they are found. Scientists also use the term somatic stem cell instead of adult stem cell, where somatic refers to cells of the body (not the germ cells, sperm or eggs). Unlike embryonic stem cells, which are defined by their origin (cells from the preimplantation-stage embryo), the origin of adult stem cells in some mature tissues is still under investigation.

Research on adult stem cells has generated a great deal of excitement. Scientists have found adult stem cells in many more tissues than they once thought possible. This finding has led researchers and clinicians to ask whether adult stem cells could be used for transplants. In fact, adult hematopoietic, or blood-forming, stem cells from bone marrow have been used in transplants for more than 40 years. Scientists now have evidence that stem cells exist in the brain and the heart, two locations where adult stem cells were not at first expected to reside. If the differentiation of adult stem cells can be controlled in the laboratory, these cells may become the basis of transplantation-based therapies $[2,3]$.

The history of research on adult stem cells began more than 60 years ago. In the 1950s, researchers discovered that the bone marrow contains at least two kinds of stem cells. One population, called hematopoietic stem cells, forms all the types of blood cells in the body. A second population, called bone marrow stromal stem cells (also called mesenchymal stem cells, or skeletal stem cells by some), were discovered a few years later. These non-hematopoietic stem cells make up a small proportion of the stromal cell population in the bone marrow and can generate bone, cartilage, and fat cells that support the formation of blood and fibrous connective tissue.
In the 1960s, scientists who were studying rats discovered two regions of the brain that contained dividing cells that ultimately become nerve cells. Despite these reports, most scientists believed that the adult brain could not generate new nerve cells. It was not until the 1990s that scientists agreed that the adult brain does contain stem cells that are able to generate the brain's three major cell types-astrocytes and oligodendrocytes, which are non-neuronal cells, and neurons, or nerve cells.

\section{Where are adult stem cells found, and what do they normally do?}

Adult stem cells have been identified in many organs and tissues, including brain, bone marrow, peripheral blood, blood vessels, skeletal muscle, skin, teeth, heart, gut, liver, ovarian epithelium, and testis. They are thought to reside in a specific area of each tissue (called a "stem cell niche"). In many tissues, current evidence suggests that some types of stem cells are pericytes, cells that compose the outermost layer of small blood vessels. Stem cells may remain quiescent (non-dividing) for long periods of time until they are activated by a normal need for more cells to maintain tissues, or by disease or tissue injury [3].

Typically, there is a very small number of stem cells in each tissue and, once removed from the body, their capacity to divide is limited, making generation of large quantities of stem cells difficult. Scientists in many laboratories are trying to find better ways to grow large quantities of adult stem cells in cell culture and to manipulate them to generate specific cell types so they can be used to treat injury or disease. Some examples of potential treatments include regenerating bone using cells derived from bone marrow stroma, developing insulin-producing cells for type 1 diabetes, and repairing damaged heart muscle following a heart attack with cardiac muscle cells.

\section{What are the similarities and differences between embryonic and adult stem cells?}

\begin{tabular}{|l|l|}
\hline \multicolumn{1}{|c|}{ Embryonic stem cells } & \multicolumn{1}{c|}{ Adult/Somatic stem cells } \\
\hline $\begin{array}{l}\text { Embryonic stem cells originate from embryos } \\
\text { during the first 5 days after fertilization. }\end{array}$ & $\begin{array}{l}\text { Somatic stem cells also known as adult stem } \\
\text { cells are thought to be undifferentiated cells, } \\
\text { found among differentiated cells in a tissue or } \\
\text { organ. }\end{array}$ \\
\hline $\begin{array}{l}\text { Stem cells have complete and total potential to } \\
\text { differentiate into any cell and that is why they } \\
\text { are called "Totipotent Stem Cells" }\end{array}$ & $\begin{array}{l}\text { Usually somatic stem cells can only give rise } \\
\text { to the cells of that tissue. Known as } \\
\text { multipotent, they have the potential to be any } \\
\text { cell in that specific tissue or organ but not a } \\
\text { cell of any other tissue or organ }\end{array}$ \\
\hline & \\
\hline
\end{tabular}

Transdifferentiation: A number of experiments have reported that certain adult stem cell types can differentiate into cell types seen in organs or tissues other than those expected from the cells' predicted lineage (i.e., brain stem cells that differentiate into blood cells or bloodforming cells that differentiate into cardiac muscle cells, and so forth). This reported phenomenon is called transdifferentiation.

\section{What are induced pluripotent stem cells?}

Induced pluripotent stem cells (iPSCs) are adult cells that have been genetically reprogrammed to an embryonic stem cell-like state by being forced to express genes and factors important for maintaining the defining properties of embryonic stem cells [4]. 


\section{Veterinary applications}

Research currently conducted on horses, dogs, and cats can benefit the development of stem cell treatments in veterinary medicine and can target a wide range of injuries and diseases such as myocardial infarction, stroke, tendon and ligament damage, osteoarthritis, osteochondrosis and muscular dystrophy both in large animals, as well as humans. While investigation of cell-based therapeutics generally reflects human medical needs, the high degree of frequency and severity of certain injuries in racehorses has put veterinary medicine at the forefront of this novel regenerative approach. Companion animals can serve as clinically relevant models that closely mimic human disease.

\section{Development of regenerative treatment models}

Stem cells are thought to mediate repair via five primary mechanisms:

1) providing an anti-inflammatory effect

2) homing to damaged tissues and recruiting other cells, such as endothelial progenitor cells, that are necessary for tissue growth

3) supporting tissue remodeling over scar formation

4) inhibiting apoptosis

5) differentiating into bone, cartilage, tendon, and ligament tissue.

To further enrich blood supply to the damaged areas, and consequently promote tissue regeneration, platelet-rich plasma could be used in conjunction with stem cell transplantation.

The efficacy of some stem cell populations may also be affected by the method of delivery; for instance, to regenerate bone, stem cells are often introduced in a scaffold where they produce the minerals necessary for generation of functional bone.

Stem cells have also been shown to have a low immunogenicity due to the relatively low number of MHC molecules found on their surface. In addition, they have been found to secrete chemokines that alter the immune response and promote tolerance of the new tissue. This allows for allogeneic treatments to be performed without a high rejection risk.

\section{Sources of stem cells}

Veterinary applications of stem cell therapy as a means of tissue regeneration have been largely shaped by research that began with the use of adult-derived mesenchymal stem cells to treat animals with injuries or defects affecting bone, cartilage, ligaments and/or tendons. There are two main categories of stem cells used for treatments: allogeneic stem cells derived from a genetically different donor within the same species and autologous mesenchymal stem cells, derived from the patient prior to use in various treatments. A third category, xenogenic stem cells, or stem cells derived from different species, are used primarily for research purposes, especially for human treatments.

Most stem cells intended for regenerative therapy are generally isolated either from the patient's bone marrow or from adipose tissue. Mesenchymal stem cells can differentiate into the cells that make up bone, cartilage, tendons, and ligaments, as well as muscle, neural and other progenitor tissues, they have been the main type of stem cells studied in the treatment of diseases affecting these tissues. The number of stem cells transplanted into damaged tissue may alter efficacy of treatment. Accordingly, stem cells derived from bone marrow aspirates, for instance, are cultured in specialized laboratories for expansion to millions of cells. Although adipose derived tissue also requires processing prior to use, the culturing methodology for adipose derived stem cells is not as extensive as that for bone marrowderived cells. While it is thought that bone-marrow derived stem cells are preferred for bone, cartilage, ligament, and tendon repair, others believe that the less challenging collection techniques and the multicellular microenvironment already present in adipose-derived stem cell fractions make the latter the preferred source for autologous transplantation.

New sources of mesenchymal stem cells are currently being researched, including stem cells present in the skin and dermis which are of interest because of the ease at which they can be harvested with minimal risk to the animal. Hematopoetic stem cells have also been discovered to be travelling in the blood stream and possess equal differentiating ability as other mesenchymal stem cells, again with a very non-invasive harvesting technique. There has been more recent interest in the use of extra embryonic mesenchymal stem cells. Research is currently underway to examine the differentiating capabilities of stem cells found in the umbilical cord, yolk sac and placenta of different animals. These stem cells are thought to have more differentiating ability than their adult counterparts, including the ability to more readily form tissues of endodermal and ectodermal origin.

\section{Stem cells and hard tissue repair}

Because of the general positive healing capabilities of stem cells, they have gained interest for the treatment of cutaneous wounds. This is important interest for those with reduced healing capabilities, like diabetics and those undergoing chemotherapy. In one trial, stem cells were isolated from the Wharton's jelly of the umbilical cord. These cells were injected directly into the wounds. Within a week, full reepithelialization of the wounds had occurred, compared to minor reepithelialization in the control wounds. This also showed the capabilities of mesenchymal stem cells in the repair of epidermal tissues.

Soft palate defects in horses are caused by a failure of the embryo to fully close at the midline during embryogenesis. These are often not found until after they have become worse because of the difficulty in visualizing the entire soft palate. This lack of visualization is thought to also contribute to the low success rate in surgical intervention to repair the defect. As a result, the horse often has to be euthanized. Recently, the use of mesenchymal stem cells has been added to the conventional treatments. After the surgeon has sutured the palate closed, autologous mesenchymal cells are injected into the soft palate. The stem cells were found to be integrated into the healing tissue especially along the border with the old tissue. There was also a large reduction in the number of inflammatory cells present, which is thought to aid in the healing process [5].

\section{Stem cells and orthopedic repairs}

Autologous stem cell-based treatments for ligament injury, tendon injury, osteoarthritis, osteochondrosis, and sub-chondral bone cysts have been commercially available to practicing veterinarians to treat horses since 2003 in the United States and since 2006 in the United Kingdom. Autologous stem cell based treatments for tendon injury, ligament injury, and osteoarthritis in dogs have been available to veterinarians in the United States since 2005. Over 3000 privately owned horses and dogs have been treated with autologous adiposederived stem cells. The efficacy of these treatments has been shown in double-blind clinical trials for dogs with osteoarthritis of the hip and elbow and horses with tendon damage [6]. 


\section{Tendon repair}

Race horses are especially prone to injuries of the tendon and ligaments. Conventional therapies are very unsuccessful in returning the horse to full functioning potential. Natural healing, guided by the conventional treatments, leads to the formation of fibrous scar tissue that reduces flexibility and full joint movement. Traditional treatments prevented a large number of horses from returning to full activity and also have a high incidence of re-injury due to the stiff nature of the scarred tendon. Introduction of both bone marrow and adipose derived stem cells, along with natural mechanical stimulus promoted the regeneration of tendon tissue. The natural movement promoted the alignment of the new fibers and tendocytes with the natural alignment found in uninjured tendons. Stem cell treatment not only allowed more horses to return to full duty and also greatly reduced the re-injury rate over a three-year period [6].

More recently, the use of embryonic stem cells has also been applied to tendon repair. The embryonic stem cells were shown to have a better survival rate in the tendon as well as better migrating capabilities to reach all areas of damaged tendon. The overall repair quality was also higher, with better tendon architecture and collagen formed. There was also no tumor formation seen during the three month experimental period. Long term studies need to be carried out to examine the long term efficacy and risks associated with the use of embryonic stem cells. Similar results have been found in animals [7].

\section{Joint repair}

Osteoarthritis is the main cause of joint pain both in animals and humans. Horses and dogs are most frequently affected arthritis. Natural cartilage regeneration is very limited and no current drug therapies are curative, but rather look to reduce the symptoms associated with the degeneration. Different types of mesenchymal stem cells and other additives are still being researched to find the best type of cell and method for long term treatment [8].

Adipose-derived mesenchymal cells are currently the most often used because of the noninvasive harvesting. There has been a lot of success recently injecting mesenchymal stem cells directly into the joint. This is a recently developed, non-invasive technique developed for easier clinical use. Dogs receiving this treatment showed greater flexibility in their joints and less pain.

\section{Bone defect repair}

While further studies are necessary to fully characterize the use of cell-based therapeutics for treatment of bone fractures. Bone has a unique and well documented natural healing process that normally is sufficient to repair fractures and other common injuries. Misaligned breaks due to severe trauma, as well as things like tumor resections of bone cancer, are prone to improper healing if left to the natural process alone. Scaffolds composed of natural and artificial components are seeded with mesenchymal stem cells and placed in the defect. Within four weeks of placing the scaffold, newly formed bone begins to integrate with the old bone and within 32 weeks, full union is achieved. Further studies are necessary to fully characterize the use of cell-based therapeutics for treatment of bone fractures [9].

Stem cells have also been used to treat degenerative bone diseases. The normally recommended treatment for dogs that have LeggCalve-Perthes disease is to remove the head of the femur after the degeneration has progressed. Recently, mesenchymal stem cells have been injected directly in to the head of the femur, with success not only in bone regeneration, but also in pain reduction.

\section{Stem cells and muscle repairs}

Stem cells have successfully been used to ameliorate healing in the heart after myocardial infarction in dogs. Adipose and bone marrow derived stem cells were removed and induced to a cardiac cell fate before being injected into the heart. The heart was found to have improved contractility and a reduction in the damaged area four weeks after the stem cells were applied. A different trial is underway for a patch made of a porous substance on to which the stem cells are "seeded" in order to induce tissue regeneration in heart defects. Tissue was regenerated and the patch was well incorporated into the heart tissue. This is thought to be due, in part, to improved angiogenesis and reduction of inflammation. Although cardiomyocytes were produced from the mesenchymal stem cells, they did not appear to be contractile. Other treatments that induced a cardiac fate in the cells before transplanting had greater success at creating contractile heart tissue.

Stem cells have been used recently to hasten the healing of skeletal muscle tears in working dogs. Traditional therapies rely on rest and anti-inflammatory treatments have been shown to permanently reduce the functioning in the muscle. Adipose derived stem cells were injected directly into the tear of the semitendinosus muscle. Physical therapy was also completed during the healing process. Healing was observed much earlier than with traditional methods and the dogs were able to return to full work without any long term muscle damage.

\section{Stem cells and nervous system repairs}

Spinal cord injuries are one of the most common traumas brought into veterinary hospitals. Spinal injuries occur in two ways after the trauma: the primary mechanical damage, and in secondary processes, like inflammation and scar formation, in the days following the trauma. These cells involved in the secondary damage response secrete factors that promote scar formation and inhibit cellular regeneration. Mesenchymal stem cells that are induced to a neural cell fate are loaded on to a porous scaffold and are then implanted at the site of injury. The cells and scaffold secrete factors that counteract those secreted by scar forming cells and promote neural regeneration. Eight weeks later, dogs treated with stem cells showed immense improvement over those treated with conventional therapies. Dogs treated with stem cells were able to occasionally support their own weight, which has not been seen in dogs undergoing conventional therapies [10].

Treatments are also in clinical trials to repair and regenerate peripheral nerves. Peripheral nerves are more likely to be damaged, but the effects of the damage are not as widespread as seen in injuries to the spinal cord. Treatments are currently in clinical trials to repair severed nerves, with early success. Stem cells induced to a neural fate injected in to a severed nerve. Within four weeks, regeneration of previously damaged stem cells and completely formed nerve bundles were observed.

Stem cells are also in clinical phases for treatment in ophthalmology. Hematopoietic stem cells have been used to treat corneal ulcers of different origin of several horses. These ulcers were resistant to conventional treatments available, but quickly responded positively to the stem cell treatment. Stem cells were also able to restore sight in one eye of a horse with retinal detachment, allowing the horse to return to daily activities.

\section{References}

1. National Institutes of Health (U.S.) (2006) Stem cell basics [Stem cell information] 
2. Kumar S, Singh NP (2006) Stem cells: A new paradigm. Indian J Human Genetics 12: $1-6$.

3. Gupta BD (2009) An introduction to stem cells and debate surrounding them. Indian Acad Forensic Med 31: 3

4. Xiao J, Yang R, Biswas S, Qin X, Zhang M, et al. (2015) Mesenchymal Stem Cells and Induced Pluripotent Stem Cells as Therapies for Multiple Sclerosis. Int J Mol Sci 16: 9283-9302.

5. Gurudutta GU, Satija NK, Singh VK, Verma YK, Tripathi RP (2012) Stem cell therapy: a novel and futuristic treatment modality for disaster injuries. Indian J Med Res 135 : 15-25. [Crossref]
6. Schmitt A, Griensven MV, Imhoff AB, Buchmann S (2012) Application of Stem Cells in Orthopedics. Stem Cells Int 1:1-12.

7. Bullough R, Finnigan T, Kay A, Maffulli N, Forsyth NR (2008) Tendon repair through stem cell intervention: cellular and molecular approaches. Disabil Rehabil 30: 17461751. [Crossref]

8. Barry F, Murphy M (2013) Mesenchymal stem cells in joint disease and repair. Nature Rev Rheumatol 9: 584-594

9. Pagni G, Kaigler D, Rasperini G, Avila-Ortiz G, Bartel R, et al. (2012) Bone repair cells for craniofacial regeneration. Advanced Drug Delivery Reviews 64: 1310-1319.

10. Gage FH, Temple S (2013) Neural Stem Cells: Generating and Regenerating the Brain. Neuron 80: 588-601.

Copyright: (2015 Ramya K. This is an open-access article distributed under the terms of the Creative Commons Attribution License, which permits unrestricted use, distribution, and reproduction in any medium, provided the original author and source are credited. 